\title{
Conditional Models for Contextual Human Motion Recognition
}

\author{
Cristian Sminchisescu*,Atul Kanaujia, Zhiguo Li, Dimitris Metaxas** \\ *TTI-C, crismin@uchicago.edu,http://ttic.uchicago.edu/ ${ }^{\sim}$ crismin $^{1}$ \\ **Rutgers University, \{kanaujia,zhli,dnm\}@cs.rutgers.edu
}

\begin{abstract}
We describe algorithms for recognizing human motion in monocular video sequences, based on discriminative Conditional Random Fields (CRF) and Maximum Entropy Markov Models (MEMM). Existing approaches to this problem typically use generative structures like the Hidden Markov Model (HMM). Therefore they have to make simplifying, often unrealistic assumptions on the conditional independence of observations given the motion class labels and cannot accommodate rich overlapping features of the observation or long-term contextual dependencies among observations at multiple timesteps. This makes them prone to myopic failures in recognizing many human motions, because even the transition between simple human activities naturally has temporal segments of ambiguity and overlap. The correct interpretation of these sequences requires more holistic, contextual decisions, where the estimate of an activity at a particular timestep could be constrained by longer windows of observations, prior and even posterior to that timestep. This would not be computationally feasible with a HMM which requires the enumeration of a number of observation sequences exponential in the size of the context window. In this work we follow a different philosophy: instead of restrictively modeling the complex image generation process - the observation, we work with models that can unrestrictedly take it as an input, hence condition on it. Conditional models like the proposed CRFs seamlessly represent contextual dependencies and have computationally attractive properties: they support efficient, exact recognition using dynamic programming, and their parameters can be learned using convex optimization. We introduce conditional graphical models as complementary tools for human motion recognition and present an extensive set of experiments that show not only how these can successfully classify diverse human activities like walking, jumping, running, picking or dancing, but also how they can discriminate among subtle motion styles like normal walks and wander walks.
\end{abstract}

Key words: Markov random fields, discriminative models, Hidden Markov Models, human motion recognition, multiclass logistic regression, feature selection,

1 Invited Submission to Computer Vision and Image Understanding, Special Issue on Modeling People: Shape, Appearance, Movement and Behavior

Preprint submitted to Elsevier Science 21 July 2006 
conditional models, optimization. 


\section{Introduction}

Solutions for robustly tracking and recognizing human motion in natural environments are important because they can provide the basic infrastructure for the advancement of several technologies that enable adaptive visual assistants for intelligent human-computer interfaces or systems for entertainment, surveillance and security. Human tracking is complex due to the large variability in the shape and articulation of the human body, the presence of clothing or fast motions. Highly variable lighting conditions or occlusion from other people or objects further complicate the problem.

Human activity and behavior recognition, on the other hand, is challenging because human motion lacks a clear categorical structure: the motion can be often classified into several categories simultaneously, because some activities have a natural compositional (or concurrent) structure in terms of basic action units (run and hand-wave, walk and shake hands while involved in a conversation with somebody known) and because even the transition between simple activities naturally has temporal segments of ambiguity and overlap. The human motion often displays multiple levels of increasing complexity that range from action-units to activities and behaviors. The more complex the human behavior, the more difficult it becomes to perform recognition in isolation. Motions can happen at various timescales and because they often exhibit long-term dependencies, long contexts of observations may need to be considered for correct classification at particular timesteps. For instance, the motion class at a current timestep may be hard to predict using only the previous state and the current image observation alone, but may be less ambiguous if several neighboring states or observations possibly both backward and forward in time are considered. However, this computation would be hard to perform using a Hidden Markov Model (HMM) [1] where stringent independence assumptions among observations are required in order to ensure computational tractability (notably, the conditional independence of observations given the hidden class labels).

For concreteness, consider a person doing a turn during dancing. After observing several image frames, the nature of this activity may become clear. However, initially, a turn followed by an ample arm movement may be very similar to the beginning of a conversation. Making local decisions for the current state constrained by a longer observation context, both prior and posterior in time can be critical for increasing the confidence (hence accuracy) of the inferred activity percept. A clear illustration is given in our fig. 6. A model that myopically decides the current motion class based on the previous state and the current observation only achieves $56 \%$ recognition accuracy for dancing. The remaining test instances are misclassified as conversation. In turn, a flexible conditional model that decides based on the previous state label and 
a contextual window of 7 observtions centered at the current state to be labeled achieves $100 \%$ recognition accuracy and has a large recognition margin w.r.t. to the other activities in its repertoire. The importance of context is also apparent whenever one has to make subtle distinctions between human activity styles, e.g. normal walking, wander walking or slow walking. These styles contain many similar $3 \mathrm{~d}$ poses with similar image appearance. Constraining the style estimate at each timestep based on a long-range context of prior observations can significantly improve its recognition performance. See our fig. 5 and fig. 7 as well as tables $4-5$.

To summarize, HMMs and more generally the class of stochastic grammars, are generative models that define a joint probability distribution $p(\mathbf{X}, \mathbf{R})$ over observations $\mathbf{R}$ and motion label sequences $\mathbf{X}$ and use Bayes rule to compute $p(\mathbf{X} \mid \mathbf{R})$. In order to model the observation process and enumerate all possible sequences of observations, generative models need to assume them atomic and independent. Therefore they can't accommodate multiple overlapping features of the observation or long-range dependencies between state indexes and observation indexes at multiple time steps because the inference problem in this case becomes intractable. Arguably, another inconvenient of using generative models like HMMs stems from their indirection: they use a joint probabilistic model to solve a conditional inference problem thus focusing on modeling the observations that at runtime are fixed. Even if the generative model were accurate, this approach could be non-economical in cases where the underlying generative model may be quite complex but the motion class conditioned on the observation (or the boundary between classes) is nevertheless simple.

In this paper we advocate a complementary discriminative approach to human motion recognition based on extensions to Conditional Random Fields (CRF) [2] and Maximum Entropy Markov Models (MEMM) [3]. A CRF conditions on the observation without modeling it, therefore it avoids independence assumptions and can accommodate long range interactions between observations and states at different timesteps. Our approach is based on non-locally defined, multiple features of the observation, represented as log-linear models - it can be viewed as a generalization of logistic regression to account for correlations among successive class labels. Inference can be performed efficiently using dynamic programming, whereas training the parameters is based on a convex problem, with guaranteed global optimality. We demonstrate the algorithms on the task of both recognizing broader classes of human motions like walking, running, jumping, conversation or dancing, but also for finely discriminating among motion styles like slow walk or wander walk. We compare against HMMs and demonstrate that the conditional models can significantly improve recognition performance in tests that use both features extracted from $3 \mathrm{~d}$ reconstructed joint angles, but also in recognition experiments that use feature descriptors extracted directly from image silhouettes. Combining the relative advantages of generative and conditional models for robust recog- 
nition remains a promising avenue for future work.

Paper Organization: $\S 1.1$ discusses related work, $\S 2$ introduces directed and undirected recognition models and discusses their relative trade-offs, $\S 3$ describes experimental results on human motion recognition. The proposed CRF and MEMM models are compared to HMMs in two series of examples, one using $2 \mathrm{~d}$ image features and the other using $3 \mathrm{~d}$ joint angle features, on both real and artificial image sequences. In section $\S 4$ we conclude and discuss perspectives for future work.

\subsection{Related Work}

The research devoted to human motion recognition is extensive, accounting for its clear social and technological importance. We refer to the comprehensive reviews by Aggarwal \& Cai [4], Gavrila [5], Pavlovic et al [6] or Hu et al [7], and here only aim at a limited literature overview. Approaches to human activity recognition can be both supervised [8,1,9-13] and unsupervised [14-17]. The methods can be subdivided into: $(i)$ Techniques based on spatiotemporal features extract templates for each activity and match them to those of unseen events $[18,19,17,14]$; (ii) Methods based on temporal logic represent critical ordering constraints on activities [20,21], and (iii) Algorithms based on stochastic grammars or Hidden Markov Models (HMM) represent the observed data distribution and model the dynamic, temporal state constraints $[9-13,22]$.

HMMs [1] and their various extensions have been successfully used for recognizing human motion based on both $2 \mathrm{~d}$ observations [9-12] and 3d observations [13,23]. More sophisticated recognition structures have been obtained by compositing the basic ones: $(i)$ Layering [22,24] composes recognition modules hierarchically by feeding the results of one recognition layer as observations for the next one; (ii) Left-right modeling allows certain transitions, but not all the possible ones between different class labels: as time increases, the state index only increases or remains the same; (iii) Factorial methods [25] use variational inference to decouple dependencies in models with multivariate discrete states. To make state inference tractable, some of the correlations between the different dimensions are relaxed. The computation is performed independently on the temporal chains corresponding to each one of them. (iv) Parallel techniques [13] recognize using a battery of models independently trained for each different action in a corpus. The most probable motion class is decided based on model selection procedures; $(v)$ Coupling [10] operates using a number of parallel HMMs where each individual state dimension depends on all the other ones. All these constructions can also be used within the framework of conditional modeling proposed in this paper. 
Generative approaches to simultaneous (mixed) tracking and motion classification have been proposed by Blake et al [26] and Pavlovic \& Rehg [27]. In their algorithms, the variability within each motion class is represented as an auto-regressive process, or a linear dynamical system, with learning and inference based on Condensation and variational techniques, respectively. Black \& Jepson [28] model motion as a trajectory through a state space spanned by optical flow fields and infer the activity class based on propagating multiple hypotheses using a Condensation filter. Fablet \& Bouthemy [8] present a powerful approach to recognition using multiscale Gibbs models. Shi et al [29] employ a propagation network and a discrete Condensation algorithm in order to better take into account sequential activities that include parallel streams of action. Fanti et al [30] represent the human motion dependencies by a triangulated graph and exploit position, velocity and appearance cues within a recognition procedure based on belief propagation. Vasilescu [16] represents human motion signatures w.r.t. as projection on a multilinear basis and classify using nearest neighbor. Hoey \& Little [31] recognize human motion using HMMs having flow field observations represented w.r.t. to a Zernike basis. Yilmaz \& Shah [32] recognize human motion using multiple uncalibrated cameras and a criteria based on the condition number of a matrix that stores $3 \mathrm{~d}$ reconstructed points on the human body. Efros et al [17] represent motion using descriptors based on optical flow with rectification and blurring and recognize actions using nearest neighbor schemes. Manor \& Irani [14] represent events using feature histograms at multiple temporal scales and compare different actions using a histogram measure based on $\mathcal{X}^{2}$ divergence.

We are not aware of conditional approaches previously applied to human motion recognition (temporal chains) but discriminative models have been successfully demonstrated in spatial inference, for the propose of detecting manmade or natural structures in images [33-36]. CRFs and MEMMs are also related to 'sliding window' methods [37] that predict each state label of a sequence independently by classifying it based on a window of observations (forward and backward in time) centered at the current state. However such methods do not account for correlations between neighboring, temporal state labels, as common in motion recognition problems.

\section{Conditional Models for Recognition}

We work with graphical models with a linear chain structure, as shown in fig. 1. These have discrete temporal states $x_{t}$, here discrete motion class labels $x \in$ $\mathcal{X}=\{1,2, \ldots, c\}, t=1 \ldots T$, prior $p\left(x_{1}\right)$, observations $\mathbf{r}_{t}$, with $\operatorname{dim}(\mathbf{r})=r$. For notational compactness, we also consider joint states $\mathbf{X}_{t}=\left(x_{1}, x_{2}, \ldots, x_{t}\right)$ or joint observations $\mathbf{R}_{t}=\left(\mathbf{r}_{1}, \ldots, \mathbf{r}_{t}\right)$. Occasionally we drop the subscript, i.e. $\mathbf{X}_{T}=\mathbf{X}$ and $\mathbf{R}_{T}=\mathbf{R}$, for brevity. 


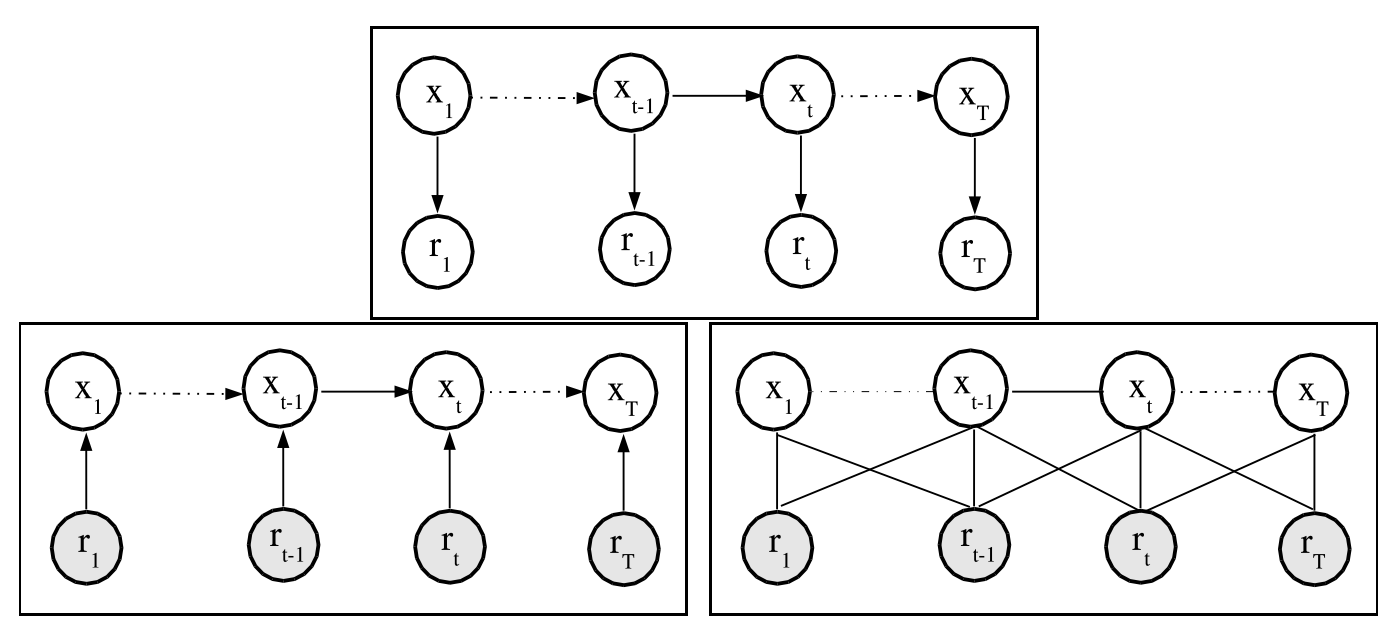

Fig. 1. (a, Top) A generative Hidden Markov Model models the observation $p\left(\mathbf{r}_{t} \mid x_{t}\right)$ and the state dynamics $p\left(x_{t} \mid x_{t-1}\right)$ and requires a probabilistic inversion to compute $p\left(\mathbf{X}_{T} \mid \mathbf{R}_{T}\right)$ using Bayes rule. Modeling the observation in terms of overlapping features or modeling long range dependencies between temporal observations and states is not tractable. (b, Bottom-left) A directed conditional model (e.g. a Maximum Entropy Markov Model) represents $p\left(x_{t} \mid x_{t-1}, r_{t}\right)$ or, more generally, a locally normalized conditional distribution based on the previous state, and a past observation window of arbitrary size. Shadowed nodes indicate that the model conditions on the observation without modeling it. But the local normalization may face label-bias problems (see text). (c, Bottom-right) A Conditional Random Field accommodates arbitrary overlapping features of the observation. Here we show a model based on a context of 3 observation timesteps, but the dependencies can be arbitrarily long-range. Generally, the architecture does not rule out an on-line system where long-range dependencies from the current state can be restricted only towards past observations. For human motion understanding, analysis based on longer observation contexts is critical for correctly resolving locally ambiguous activity classes. For instance consider the case when one initiates a conversation by vividly moving her arms. This may well be labeled as a conversation but it may also initially look like dancing. Consider also the subtle distinctions between normal walks, wander walks or slow walks. These activities may contain very similar individual poses and only the analysis of long-term dynamic dependencies in the observation sequence may help identify the correct class. See our fig. 5 and fig. 7 as well as tables $4-5$.

\subsection{Undirected Models. Conditional Random Fields}

Let $G=(V, E)$ be a graph and $\mathbf{X}$ being indexed by the vertices of $G$, say $x_{i}$. A pair $(\mathbf{X}, \mathbf{R})$ is called a Conditional Random Field (CRF) [2], if when conditioning on $\mathbf{R}$, the variables $x_{i}$ obey the Markov property w.r.t. the graph: $p\left(x_{i} \mid \mathbf{R}, \mathbf{X}_{V-\{i\}}\right)=p\left(x_{i} \mid \mathbf{R}, \mathbf{X}_{\mathcal{N}_{i}}\right)$, where $\mathcal{N}_{i}$ is the set of neighbors of node $i$ and $\mathbf{X}_{\mathcal{N}_{i}}$ is the joint vector of variables in the subscript set. Let $C(\mathbf{X}, \mathbf{R})$ be the set of maximal cliques of $G$. Using the Hammersley Clifford theorem [38], the distribution over joint labels $\mathbf{X}$ given observations $\mathbf{R}$ and parameters $\boldsymbol{\theta}$, can be written as an expansion: 


$$
p_{\boldsymbol{\theta}}(\mathbf{X} \mid \mathbf{R})=\frac{1}{Z_{\boldsymbol{\theta}}(\mathbf{R})} \prod_{c \in C(\mathbf{X}, \mathbf{R})} \phi_{\boldsymbol{\theta}}^{c}\left(\mathbf{X}_{c}, \mathbf{R}_{c}\right)
$$

where $\phi_{\boldsymbol{\theta}}^{c}$ is the positive-valued potential function of clique $c$, and $Z_{\boldsymbol{\theta}}(\mathbf{R})$ is the observation dependent normalization:

$$
Z_{\boldsymbol{\theta}}(\mathbf{R})=\sum_{\mathbf{X}} \prod_{c \in C(\mathbf{X}, \mathbf{R})} \phi_{\boldsymbol{\theta}}^{c}\left(\mathbf{X}_{c}, \mathbf{R}_{c}\right)
$$

For a linear chain (first-order state dependency), the cliques include pairs of neighboring states $\left(x_{t-1}, x_{t}\right)$, whereas the connectivity among observations is unrestricted, as these are known and fixed (see fig. 1b). Therefore, arbitrary clique structures that include complex observation dependencies do not complicate inference. For a model with $T$ timesteps, the CRF in (1) can be rewritten in terms of exponentiated feature functions $F_{\boldsymbol{\theta}}$ computed in terms of weighted sums over the features of the cliques, c.f. (3) and (12): ${ }^{2}$

$$
\begin{aligned}
p_{\boldsymbol{\theta}}(\mathbf{X} \mid \mathbf{R}) & =\frac{1}{Z_{\boldsymbol{\theta}}(\mathbf{R})} \exp \left(\sum_{t=1}^{T} F_{\boldsymbol{\theta}}\left(x_{t}, x_{t-1}, \mathbf{R}\right)\right) \\
Z_{\boldsymbol{\theta}}(\mathbf{R}) & =\sum_{\mathbf{X}} \exp \left(\sum_{t=1}^{T} F_{\boldsymbol{\theta}}\left(x_{t}, x_{t-1}, \mathbf{R}\right)\right)
\end{aligned}
$$

Assuming a fully labeled training set $\left\{\mathbf{X}^{d}, \mathbf{R}^{d}\right\}_{d=1 \ldots D}$, the CRF parameters can be obtained by optimizing the conditional log-likelihood:

$$
\begin{aligned}
\mathcal{L}_{\boldsymbol{\theta}} & =\sum_{d=1}^{D} \log p_{\boldsymbol{\theta}}\left(\mathbf{X}^{d} \mid \mathbf{R}^{d}\right)= \\
& =\sum_{d=1}^{D}\left(\sum_{i=t}^{T} F_{\boldsymbol{\theta}}\left(x_{t}^{d}, x_{t-1}^{d}, \mathbf{R}^{d}\right)-\log Z_{\boldsymbol{\theta}}\left(\mathbf{R}^{d}\right)\right)
\end{aligned}
$$

In practice, we often regularize the problem by optimizing a penalized likelihood: $\mathcal{L}_{\boldsymbol{\theta}}+\mathcal{R}_{\boldsymbol{\theta}}$, either using soft (ridge) feature selection: $\mathcal{R}_{\boldsymbol{\theta}}=-\|\boldsymbol{\theta}\|^{2}$, or a more aggressive Jeffrey prior: $\mathcal{R}_{\boldsymbol{\theta}}=-\log \|\boldsymbol{\theta}\|$.

Likelihood maximization can be performed using a gradient ascent (e.g. BFGS

$\overline{2}$ We use a model with tied parameters $\boldsymbol{\theta}$ across all cliques, in order to seamlessly handle models of arbitrary size, i.e., sequences of arbitrary length. 
[39]) method:

$$
\begin{aligned}
\frac{d \mathcal{L}_{\boldsymbol{\theta}}}{\mathbf{d} \boldsymbol{\theta}}=\sum_{d=1}^{D} & \left(\sum_{t=1}^{T} \frac{d F_{\boldsymbol{\theta}}\left(x_{t}^{d}, x_{t-1}^{d}, \mathbf{R}^{d}\right)}{\mathbf{d} \boldsymbol{\theta}}-\right. \\
& \left.-\sum_{\mathbf{X}} p_{\boldsymbol{\theta}}\left(\mathbf{X} \mid \mathbf{R}^{d}\right) \sum_{t=1}^{T} \frac{d F_{\boldsymbol{\theta}}\left(x_{t}, x_{t-1}, \mathbf{R}^{d}\right)}{\mathbf{d} \boldsymbol{\theta}}\right)
\end{aligned}
$$

For discrete-valued chain models with state dependencies acting over a short range, the observation dependent normalization can be computed efficiently by matrix / tensor multiplication. For a bigram model, we work with the matrix of size $c \times c$, containing all possible assignments of pairs of neighboring states to class labels: ${ }^{3}$

$$
M_{t}(\mathbf{R})=\left[\exp \left(F_{\boldsymbol{\theta}}\left(x_{t}, x_{t-1}, \mathbf{R}\right)\right)\right], x_{t}, x_{t-1} \in \mathcal{X}
$$

Then the observation dependent normalization factor can be computed as:

$$
Z_{\boldsymbol{\theta}}(\mathbf{R})=\left(\prod_{t=1}^{T+1} M_{t}(\mathbf{R})\right)_{i n i t i a l, f i n a l}
$$

where we have added two dummy initial and final states $x_{0}=$ initial and $x_{T+1}=$ final and the subscript indicates the particular entry of the matrix product [2].

The conditional probability of a class label sequence is:

$$
p_{\boldsymbol{\theta}}(\mathbf{X} \mid \mathbf{R})=\frac{\prod_{t=1}^{T+1} \exp \left(F_{\boldsymbol{\theta}}\left(x_{t}, x_{t-1}, \mathbf{R}\right)\right)}{Z_{\boldsymbol{\theta}}(\mathbf{R})}
$$

The potential functions at pairs of neighboring sites can be chosen as:

$$
F_{\boldsymbol{\theta}}\left(x_{t}, x_{t-1}, \mathbf{R}\right)=\psi_{\boldsymbol{\theta}}\left(x_{t}, \mathbf{R}\right)+\psi_{\boldsymbol{\theta}}\left(x_{t}, x_{t-1}\right)
$$

where $\psi_{\boldsymbol{\theta}}$ are linear models:

$$
\psi_{\boldsymbol{\theta}}\left(x_{t}, \mathbf{R}\right)=\sum_{a=1}^{A} \lambda_{a} f_{a}\left(x_{t}, \mathbf{R}\right)
$$

$\overline{3}$ Longer range state interactions be accommodated, e.g., a trigram model by working with a tensor of size $c^{3}$. 


$$
\psi_{\boldsymbol{\theta}}\left(x_{t}, x_{t-1}\right)=\sum_{b=1}^{B} \beta_{b} g_{b}\left(x_{t}, x_{t-1}\right)
$$

with parameters $\boldsymbol{\theta}=\left\{\left(\lambda_{a}, \beta_{b}\right), a=1 \ldots A, b=1 \ldots B\right\}$, to be estimated, and preset feature functions $f_{a}, g_{b}$ based on conjunctions of simple rules. For instance, given a temporal context window of size $2 W+1$ (observations) around the current observation time index, the combined observation-label feature function is:

$$
f_{a}\left(x_{t}, \mathbf{R}\right)=\mathbb{I}\left[x_{t}=m\right] \mathbf{r}_{t-j}[i], \text { where } m \in \mathcal{X}, i \in\{1 \ldots r\}, j \in[-W, W]
$$

for a total of $A=c \times(2 W+1) \times r$ feature functions ( $\mathbb{I}$ is the indicator function). Intuitively, the features encode correlations among motion classes and components of the observation vector forward or backward in time. Each feature function indexed by $a \in\{1 \ldots A\}$ focuses on a particular motion class instance $m$ and a particular component of the observation vector $i$ at a time offset $j$ with respect to the current state index. Similarly, the features that model inter-class dependencies are:

$$
g_{b}\left(x_{t}, x_{t-1}\right)=\mathbb{I}\left[x_{t}=m_{1} \wedge x_{t-1}=m_{2}\right], \text { where } m_{1}, m_{2} \in \mathcal{X}
$$

for a total of $B=c^{2}$ functions.

CRFs are convenient because, as for HMMs, inference can be performed efficiently using dynamic programming. Learning the model parameters leads to a convex problem with guaranteed global optimality [2]. We solve this optimization using a limited-memory variable-metric gradient ascent (BFGS) method [39] that converges in a couple of hundred iterations in most of our experiments (see fig. 4).

\subsection{Directed Conditional Models. Maximum Entropy Markov Models (MEMM)}

An alternative approach to conditional modeling is to use a directed model [3] as shown in fig. 1b. This requires a locally normalized representation for $p\left(x_{t} \mid x_{t-1}, \mathbf{r}_{t}\right)$. Inference can be performed efficiently using a dynamic programming procedure based on recursive Viterbi steps:

$$
\alpha_{t}(x)=\sum_{x^{\prime} \in \mathcal{X}} \alpha_{t-1}(x) \cdot p\left(x \mid x^{\prime}, \mathbf{r}_{t}\right)
$$

where $\alpha_{t}(x)$ computes the probability of being in state $x$ at time $t$, given the observation sequence up to time $t$. Similarly, the backward procedure computes $\beta_{t}$ as the probability of starting from state $x$ at time $t$, given the observation 
sequence after time $t$ as:

$$
\beta_{t}\left(x^{\prime}\right)=\sum_{x \in \mathcal{X}} p\left(x \mid x^{\prime}, \mathbf{r}_{t}\right) \cdot \beta_{t+1}(x)
$$

The conditional distribution $p\left(x_{t} \mid x_{t-1}, \mathbf{r}_{t}\right)$ can be modeled as a log-linear model expressed in terms of feature functions $F_{\boldsymbol{\theta}}$ as in (12), (13) and (14):

$$
p\left(x_{t} \mid x_{t-1}, \mathbf{r}_{t}\right)=\frac{1}{Z\left(x_{t-1}, \mathbf{r}_{t}\right)} \exp \left(F_{\boldsymbol{\theta}}\left(x_{t}, x_{t-1}, \mathbf{r}_{t}\right)\right)
$$

where

$$
Z\left(x_{t-1}, \mathbf{r}_{t}\right)=\sum_{x_{t}} F_{\boldsymbol{\theta}}\left(x_{t}, x_{t-1}, \mathbf{r}_{t}\right)
$$

It is worth noticing that CRFs solve a problem that exists in MEMMs [3,2], called the label-bias problem. This problem arises because such models are locally normalized. (MEMMs still have a non-linear decision surface because the local normalization depends on the state.) The per-state normalization requirement implies that the current observation is only able to select what successor state is selected, but not the probability mass transfered to it, causing biases towards states with low-entropy transitions. In the limit, the current observation is effectively ignored for states with single outgoing transitions. In order to avoid this effect, a CRF employs an undirected graphical model that defines a single log-linear distribution over the joint vector of an entire class label sequence given a particular observation sequence (thus the model has a linear decision surface). By virtue of the global normalization, entire state sequences are interpreted jointly, and this allows individual states to boost or damp the probability mass transfered to their successive states.

\section{Experiments}

We run a variety of recognition experiments, where we compare the proposed MEMM and CRF models (with different windows of observations encoding different degrees of context) with HMMs. We do two series of tests, one using $2 \mathrm{~d}$ image features and the other using $3 \mathrm{~d}$ human joint angle features. The recognition models and algorithms used are the same in both cases, the only difference is the type of information represented in the observation vector (one series of tests use $2 \mathrm{~d}$ information whereas the other uses $3 \mathrm{~d}$ information). We report recognition accuracy as the percentage of input frames classified with the correct activity type.

Training Set: To gather image training data, we use Maya (Alias Wavefront), with realistically rendered computer graphics human surface models that we 
animate using human motion capture [40]. This database is annotated by activity class (with each individual sequence supplementary sub-segmented by activity type) and this information can be used to generate a labeled training set on which we perform segmentation and classification. Our 3d human state representation is based on an articulated skeleton with spherical joints, and has 56 d.o.f. including global translation. Our database consists of 8000 samples (individual image or 3d joint angle observations, paired with their class labels) from 10 different human activities: conversation, dance, bending forward, bending sideways, washing windows, jumping, walking (normal and wander), turning, running. For each action we acquired samples from 3 different subjects, 2 male and 1 female. We test on 3 different subjects. Some insight into the structure of the database is given in fig. 2, whereas image samples from our motion test sequences are shown in fig. 3 .

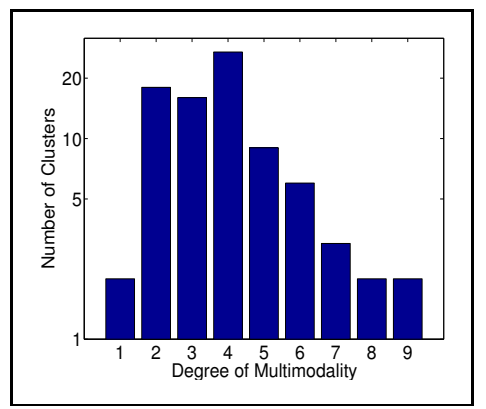

Fig. 2. Analysis of the degree of ambiguity in the motion class labeling for our database, under moderate input (silhouette) perturbations. We cluster the input silhouette feature vectors into 80 clusters, count the number of different motion labels that fall within each, and histogram those.
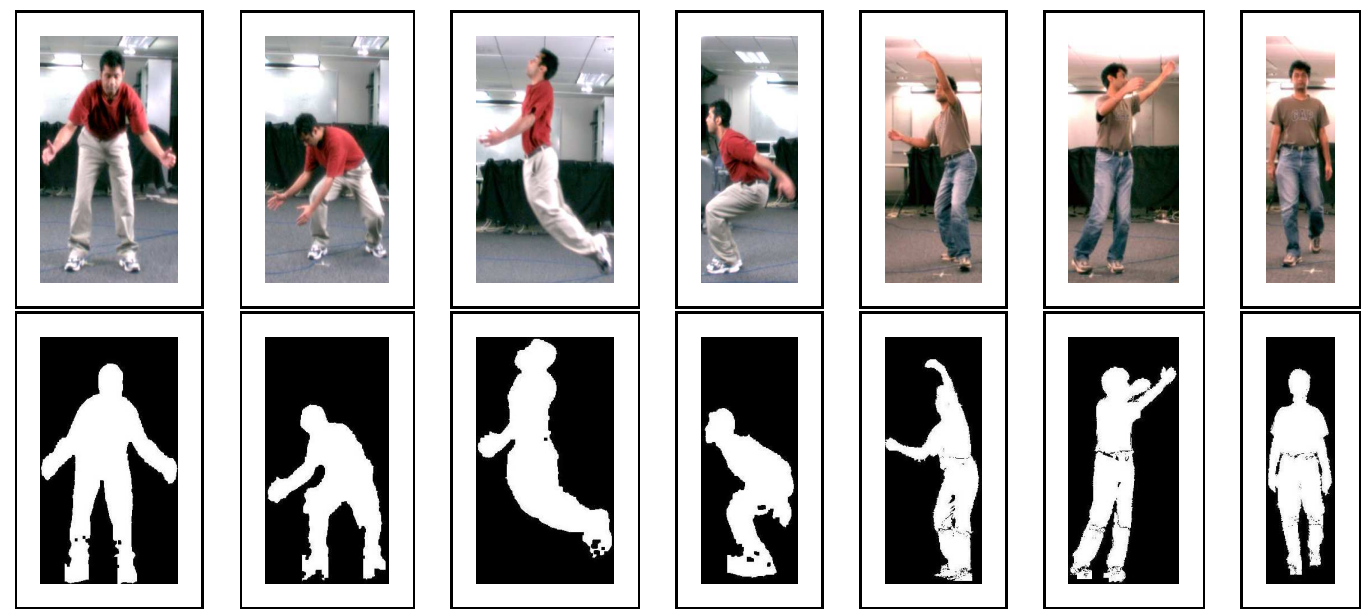

Fig. 3. Sample images (top row) and silhouettes (bottom row) of typical motions that are recognized: walking, running, bending and picking, dancing, etc. Notice that the silhouettes are of average quality and potentially quite different from the ones we trained on and which were synthetically generated. Nevertheless, we observe that the recognition accuracy is usually robust to these factors.

2d Image Features: For the image-based experiments, we work with silhou- 
ettes that we obtain using a combination of statistical background subtraction and motion segmentation [41]. As image descriptors, we use 50-dimensional histograms of combined shape context and pair-wise edge features extracted at a variety of scales on the silhouette [41]. This representation is semi-local, rich and has been effectively demonstrated in many applications, including texture recognition or pose prediction. The representation is based on overlapping features of the observation. Therefore the elements of the silhouette feature vector are generally not independent. However, due to its conditional structure, a CRF flexibly accommodates this representation without modeling assumption violations.

3d joint angle features: For 3d recognition experiments, we work with observation vectors consisting of 56 dimensional human joint angles obtained from a $3 \mathrm{~d}$ human motion reconstruction system.

Models, parameters and training times: All our training and testing used a standard $2 \mathrm{GHz}$ desktop $\mathrm{PC}$ and running times are given for this system. We perform tests in order to compare the CRF model described in $\S 2.1$ with HMMs and MEMMs. The number of parameters in the models is determined by the number of activities $c=10$, the dimension of the observation vector $\mathbf{r}$, in our case $\operatorname{dim}(\mathbf{r})=50$ for $2 \mathrm{~d}$ image features and $\operatorname{dim}(\mathbf{r})=56$ for $3 \mathrm{~d}$ human joint angle features, and the window context (for CRFs): $W=\{0,1,3\}$ implying that 0,1 or 3 observations forward and backward in time are used and the overall window size is $2 W+1$.

The HMM we use is a fully ergodic model based on Gaussian emission probabilities having diagonal covariance matrix for each state. The parameters of the model (the emission probability density, the state transition matrix) are learned from training data [1] using Maximum Likelihood (training takes about $3 \mathrm{~s})$. The total number of HMM parameters is: $2 \times c \times \operatorname{dim}(\mathbf{r})+c^{2}$ for a total of 1100 parameters in the 2d experiments and 1220 parameters for the $3 \mathrm{~d}$ experiments. We also learn a variety of CRFs that model various degrees of long-range dependencies between observations, i.e. windows $W=\{0,1,3\}$, implying that we consider contexts of observations of size 0,3 and 7 centered at the current time index. ${ }^{4}$ The total number of parameters is: $(2 W+1) \times c \times \operatorname{dim}(\mathbf{r})+c^{2}$ for a total of $600(W=1), 1600(W=3)$, $3600(W=7)$ parameters for the $2 \mathrm{~d}$ experiments and $660(W=1), 1780$

\footnotetext{
4 For the experiments, we only consider baseline models, arguably, more complex HMMs or CRFs can be used. Nevertheless, most of the technology previously used to construct sophisticated HMMs including layering or left-right models can be directly applied to build CRF counterparts (e.g. left-right implementations can be obtained by setting some of the $\beta$ parameters in (14) to zero; one can build a separate leftright model for each motion class, etc.). None of the models is thus disadvantaged by not using such features.
} 
$(W=3), 4020(W=7)$ parameters for the $3 \mathrm{~d}$ experiments. The number of parameters of the MEMMs is the same as for CRFs with $W=0$. Fig. 4 gives insight into the learning procedure for CRFs and the distribution of estimated coefficients for our feature functions (learning is convex for CRFs/MEMMs, hence parameters can be initialized to arbitrary values - we use 0). Training is more expensive for CRFs, ranging from 7 mins $(W=0), 25$ mins $(W=3)$ to 50 mins $(W=3)$. Inference is about as fast for all models in the order of 2-4 seconds for sequences of several hundred frames.
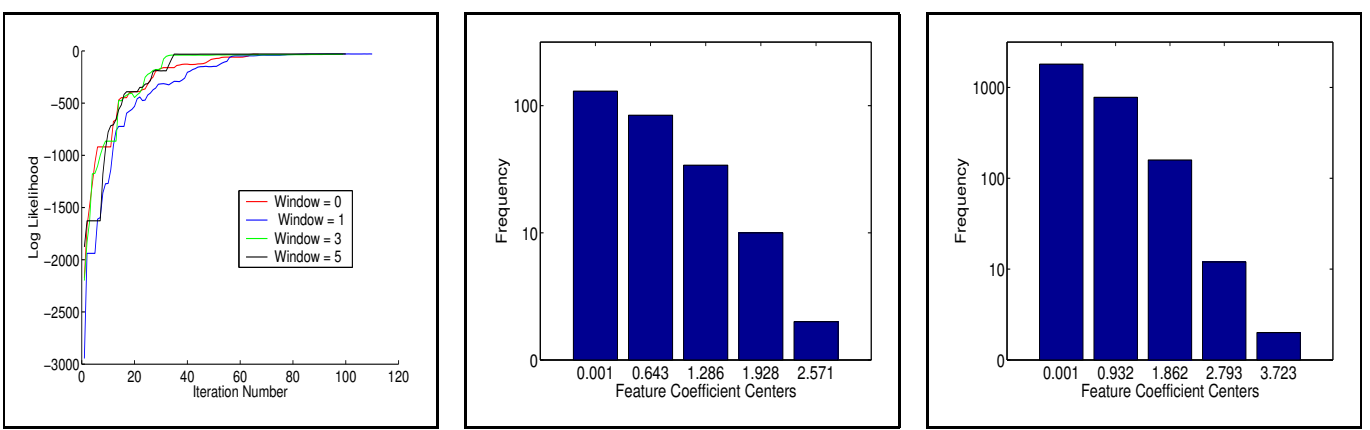

Fig. 4. (Left) plots the data conditional log-likelihood as per eq. (5), versus the iteration number for various observation windows $(W=0, \ldots, 5)$. Notice that all models converge equally fast, in about 100 iterations. (Middle) and (right) plots show histograms of parameters $\boldsymbol{\theta}$ corresponding to $W=0$ and $W=5$. Many parameters are small because we use a ridge penalized likelihood. Notice an increase in the range of parameters for models that use a larger context.

\subsection{Recognition Experiments based on 2d features}

We test our algorithms on both real data (table 2 show the results obtain by testing on 1029 frames) and artificial data (table 1 shows results obtained by testing on 2536 frames, and use a CRF with $W=1$ ) and evaluate their recognition performance not only w.r.t. broader classes of motion like running, walking or dancing, but also w.r.t. to finer styles like normal walk, wander walk or slow walk (tables $3,4,5$; we display results for 4 motion labels, we test on 700 frames and use a CRF with $W=1$ ). It is noticeable that the CRF typically outperforms the MEMM and the HMM in most test cases.

In table 2 we show an extensive set of experiments for different motion labels and models. The CRFs learned using larger window contexts generally outperform the HMM, with the exception of the jump, which the CRF confuses with the motion of picking, or of grabbing something from the floor. CRFs also show significantly better and stabler recognition performance in the presence of larger variability w.r.t. the training set (e.g. the test set denoted HWSW has input silhouettes that are significantly different from the ones on the training set). It is also important to notice how increasing the 


\begin{tabular}{|c|c|c|c|c|c|c|c|c|c|c|c||}
\hline & C & FR & FWT & JLT & PD & RLT & SR & SW & SWF & WF & WS \\
\hline CRF & 72.8 & 100 & 100 & 100 & 100 & 100 & 100 & 100 & 100 & 100 & 100 \\
\hline MEMM & 100 & 40 & 100 & 5.2 & 100 & 100 & 90.5 & 98.14 & 100 & 91.4 & 100 \\
\hline HMM & 1.4 & 100 & 2.5 & 1.7 & 87.41 & 93.75 & 100 & 100 & 100 & 100 & 100 \\
\hline
\end{tabular}

Table 1

Comparisons of recognition performance (percentage accuracy) for synthetically generated silhouette input features. $\mathrm{C}=$ Conversation, $\mathrm{FR}=$ Run seen Frontally, FWT $=$ Walk and Turn seen Frontally, JLT $=$ Jogging and Left Turn, PD = Professional Dance, RLT = Run and Turn Left, $\mathrm{SR}=$ Run seen from a Side, $\mathrm{SW}=$ Walk seen from a Side, SWF = Slow Walk seen Frontally, SWS = Slow Walk seen from a Side, $\mathrm{WF}=$ Wander walk seen Frontally, WS = Wander walk seen from a Side.

\begin{tabular}{|c|c|c|c|c|c|c|c||}
\hline & CW & D1 & D2 & BPS & LVSW & HVSW & JF \\
\hline CRF $W=0$ & 100 & 37 & 100 & 100 & 100 & 100 & 16 \\
\hline CRF $W=1$ & 100 & 42 & 96 & 100 & 100 & 100 & 27 \\
\hline CRF $W=3$ & 100 & 56.44 & 90.8 & 100 & 100 & 100 & 28 \\
\hline HMM & 100 & 39 & 90 & 76 & 98.02 & 17 & 58 \\
\hline
\end{tabular}

Table 2

Comparisons of recognition performance (percentage accuracy) for silhouettes extracted in real image sequences. $\mathrm{CW}=$ Complex Walk of a person coming towards the camera, turning and walking back, D1 = classical Dancing, D2 = modern Dancing, BPS = Bending and Picking seen from a Side, LVSW = Walking seen from a Side, silhouettes having Lower Variability w.r.t. the training set, HVSW = Walking seen from a Side, silhouettes having significantly Higher Variability w.r.t. the training set, JF = Jump Forward. The CRF with longer range dependencies generally does better, but seems to confuse the jump with the pick-up. These motions indeed have similar parts, especially given that translation information is not used in the silhouette representation (but an object centered coordinate system for features). Notice that CRF does significantly better in the presence of larger variability w.r.t. the training set (e.g. HVSW), which has been also noticed in [2].

context of the current observation improves recognition and changes the inferred distribution of class labels. In fig. 5 we show how a larger observation context can improve recognition results by as much as $70 \%$.

In tables 3, 4 and 5, we analyze the recognition performance w.r.t. viewpoint and finer motion differences. For the experiments shown in table 3, we have selected a viewpoint that is somewhat uninformative with respect to the motion. As a consequence, the recognition rates are not high, often the normal walk and the wander walk are confused.

In table 4, the recognition is generally improved (the side viewpoint appears quite informative in disambiguating running from anything else), but the MEMM and the HMM have difficulty in accounting for long-range observation dependencies that appear useful in discriminating among different 
Table 3

\begin{tabular}{|c||c|c|c|c||}
\hline & NW & WW & SW & R \\
\hline CRF $W=0$ & 38.9 & 65 & 86.5 & 100 \\
\hline CRF $W=3$ & 100 & 45 & 100 & 100 \\
\hline MEMM & 16.31 & 64.5 & 50.5 & 75 \\
\hline HMM & 0 & 76.5 & 44.3 & 100 \\
\hline
\end{tabular}

Recognition accuracy for a $45^{\circ}$ viewpoint. NW / WW / SW = Normal / Wander / Slow Walk; R = Run.

walking styles.

Table 4

\begin{tabular}{|c||c|c|c|c||}
\hline & NW & WW & SW & R \\
\hline CRF $W=0$ & 79.62 & 100 & 51 & 100 \\
\hline CRF $W=3$ & 100 & 100 & 100 & 100 \\
\hline MEMM & 59.25 & 96.57 & 53 & 100 \\
\hline HMM & 80 & 100 & 33 & 100 \\
\hline
\end{tabular}

Recognition accuracy for a side viewpoint. NW / WW / SW = Normal / Wander / Slow Walk; R = Run.

In table 5, we show recognition results for motions seen from a challenging frontal viewpoint. The wander walk tends to be the easiest to discriminate, presumably because it produces informative sideways variations in the frontally projected silhouette. CRF's contextual power helps improving performance, which nevertheless remains low, as it often confuses the normal and slow walks.

Table 5

\begin{tabular}{|c||c|c|c|c||}
\hline & NW & WW & SW & R \\
\hline CRF $W=0$ & 30.5 & 100 & 100 & 22 \\
\hline CRF $W=3$ & 36.1 & 100 & 96 & 21.5 \\
\hline MEMM & 34 & 91.5 & 96 & 16.25 \\
\hline HMM & 14.51 & 80.60 & 81 & 0 \\
\hline
\end{tabular}

Recognition accuracy for a frontal viewpoint. NW / WW / SW= Normal / Wander / Slow Walk; R = Run.

\subsection{Recognition based on reconstructed 3d joint angle features}

In table 6 we give motion recognition results based on reconstructed $3 \mathrm{~d}$ joint angle features [40], as opposed to directly based on image silhouette features (we use a variety of motion types for a total of 1200 tested frames). We directly use the human motion capture output as opposed to the $3 \mathrm{~d}$ reconstruction results from an algorithm like [41], because often multiple $3 \mathrm{~d}$ trajectories are plausible give an image sequence [42]. Therefore probabilistically correct 

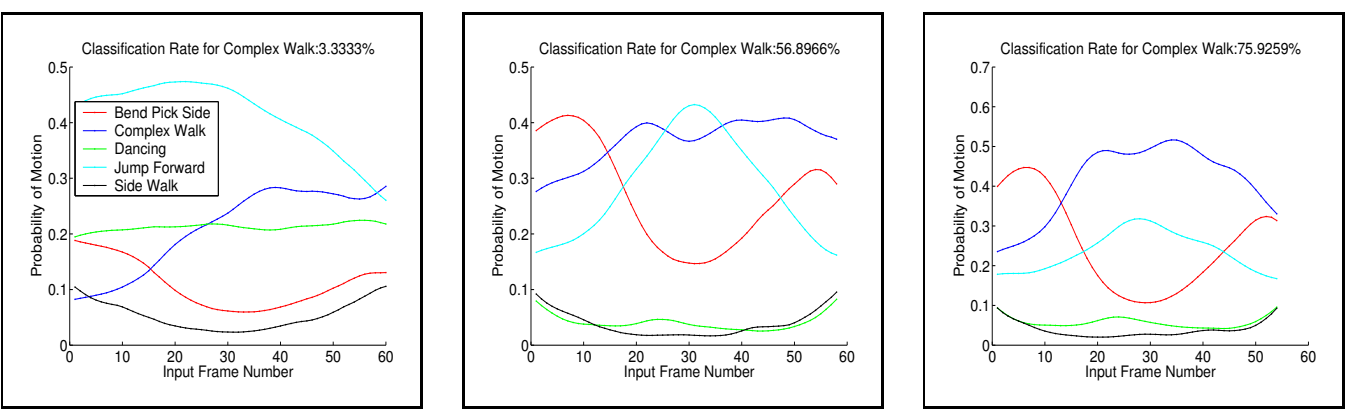

Fig. 5. (Best viewed in color) The use of context in CRFs significantly improves the recognition performance $(2 \mathrm{~d}$ testing on feature vectors based on silhouettes extracted from real images). Left plots shows the distribution over motion class labels when we use only the current observation (i.e. no context $W=0$ ) whereas the middle and right plots use contexts of size $W=1$ and $W=3$ respectively ( 3 and and 7 observation timesteps centered at the current one). A HMM tested on the same sequence entirely mis-classifies the complex walk (motion towards the camera, turning and walking back - with low accuracy of about $1.5 \%$ ), which is close to the performance of a CRF with no context (left plot).

recognition in this context would be more complex, as a recognizer may have to consider different 3d input hypotheses. The CRFs based on larger contexts have generally better performance than the HMM (see also fig. 6 and fig. 7), except for conversations which are sometimes confused with dancing (see fig. 6). This is not entirely surprising given that both of these activities involve similar, ample arm movements. The occasional drop in the performance of CRFs could be caused by insufficient training data. MEMMs can outperform CRFs in problems where their non-linear decision boundary is more adequate than the linear CRF one.

Table 6

\begin{tabular}{|c||c|c|c|c|c|c||}
\hline & R & W & SWW & RTL & C & D \\
\hline CRF $W=0$ & 100 & 100 & 0 & 100 & 60 & 56.29 \\
\hline CRF $W=3$ & 100 & 100 & 100 & 100 & 50.40 & 100 \\
\hline MEMM & 100 & 100 & 19.9 & 100 & 79.8 & 100 \\
\hline HMM & 100 & 68.5 & 0 & 100 & 82.5 & 89 \\
\hline
\end{tabular}

Recognition accuracy based on 3 d joint angle features. $\mathrm{R}=$ Running, $\mathrm{W}=$ Walking, SWW $=$ Slow Walk Wandering, RTL $=$ Run and Turn Left, $\mathrm{C}=$ Conversation, $\mathrm{D}$ $=$ Dancing. The accuracy of CRF with long-range dependencies is generally better, however it seems to confuse conversation and dancing, see also fig. 6 . This is not surprising given that both activities involve sometimes similar arm movements. Notice also how the context helped boosting the recognition performance for SWW in fig. 7. 

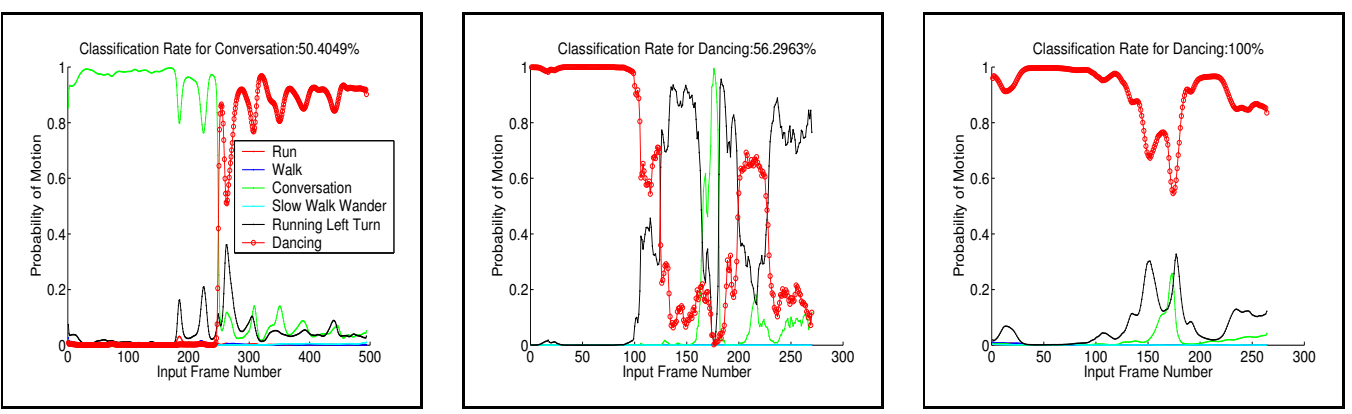

Fig. 6. (Best viewed in color) The distribution over class labels for recognition experiments based on 3d joint angle observations. Left plot shows motion class distributions for a conversation test set. Even a CRF that uses context partly confuses conversation and dancing, presumably because both classes involve similar ample arm movements. Middle shows recognition results for a dancing test sequence, based on a CRF with no context $(W=0)$. Right shows how a CRF with context $W=3$ improves the recognition performance for dancing by $43 \%$ w.r.t. the CRF with no context $(W=0$, middle $)$.
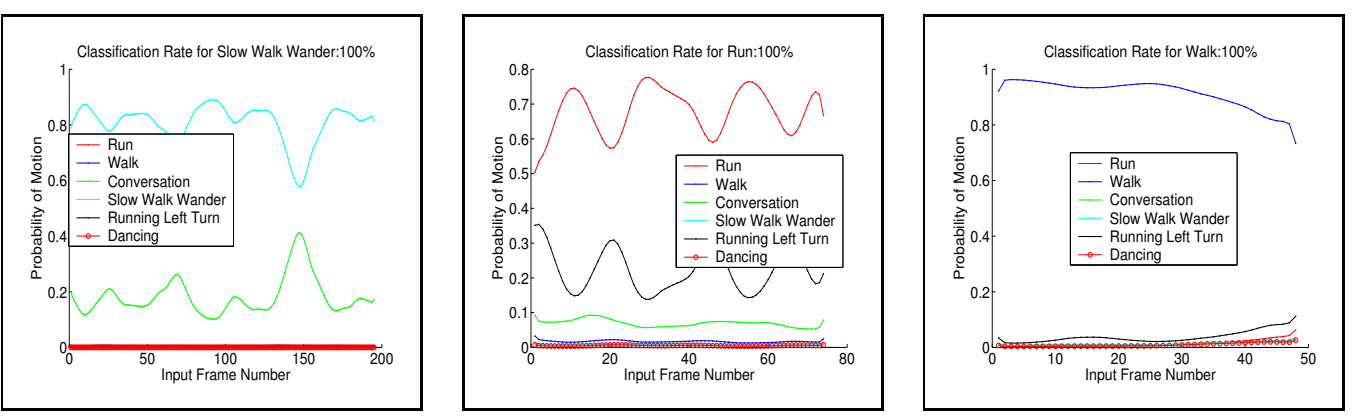

Fig. 7. (Best viewed in color) Left plot shows increased recognition performance for a slow walk wander motion, here a CRF with $W=3$ improved recognition accuracy by $100 \%$ w.r.t. to a a CRF with no context $W=0$ (see table 6). Middle and right plots show good CRF recognition accuracy for running and walking.

\section{Conclusions}

We have presented a framework for human motion recognition, that unlike existing generative approaches based on HMM, is discriminative and based on Conditional Random Fields and Maximum Entropy Markov Models. These complement the popular HMMs and can be used in tandem with them in recognition systems. By virtue of their conditional structure, the models can accommodate arbitrary overlapping features of the observation as well as longterm contextual dependencies among observations at different timesteps. This wouldn't be possible in a HMM where strict independence assumption among observations are required in order to ensure tractability. Similarly to HMMs, inference in the conditional models can be performed efficiently using dynamic programming, whereas the training procedure for the parameters is based on convex optimization. We have demonstrated the algorithms for the recognition of a variety of human motions including walking, running, bending or dancing. 
We observed that CRFs have improved recognition performance over MEMMs, that in turn, typically outperformed competing HMMs.

Future Work: Inference and learning with CRFs provides an avenue for many associated research problems. It would be interesting to systematically investigate how long-range should the observation dependency be for optimal recognition performance, as well as recognition based on different selections of features. The number of possible feature combinations can be large, so efficient methods for feature selection or feature induction are necessary. In this work we use a model with first order state dependency (a bigram) but it would be interesting to study longer range state dependencies, e.g. trigrams. All these extensions are straightforward to include in a CRF. More complex human motion recognition systems based on the ideas described here may be constructed using advanced tools based on left-right models and multiple layers. The design of models with conditional and generative subcomponents (CRF/MEMMs and HMMs) is likely to be a promising avenue for future research.

\section{Acknowledgments}

Cristian Sminchisescu gives special thanks to Allan Jepson at the University of Toronto, for many insightful discussions and feedback on the topics presented in this paper. The authors thank the anonymous reviewers for valuable comments.

\section{References}

[1] L. Rabiner, A tutorial on Hidden Markov Models and selected applications in speech recognition, Proc. IEEE 77 (2) (1989) 257-286.

[2] J. Lafferty, A. McCallum, F. Pereira, Conditional random fields: probabilistic models for segmenting and labeling sequence data, in: International Conference on Machine Learning, 2001.

[3] A. McCallum, D. Freitag, F. Pereira, Maximum entropy Markov models for information extraction and segmentation, in: International Conference on Machine Learning, 2000.

[4] J. Aggarwal, Q. Cai, Human Motion Analysis: A Review, Computer Vision and Image Understanding 73 (3) (1999) 428-440.

[5] D. Gavrila, The Visual Analysis of Human Movement: A Survey, Computer Vision and Image Understanding 73 (1) (1999) 82-98. 
[6] V. Pavlovic, R. Sharma, T. Huang, Visual interpretation of hand gestures for human-computer interaction: a review, IEEE Transactions on Pattern Analysis and Machine Intelligence.

[7] W. Hu, T. Tan, L. Wang, S. Maybank, A survey on visual surveillance of object motion and behaviours, IEEE Transactions on Systems, Man and Cybernetics.

[8] R. Fablet, P. Bouthemy, Non-parametric motion recognition using temporal multiscale Gibbs models, in: IEEE International Conference on Computer Vision and Pattern Recognition, 2001.

[9] T. Starner, A. Pentland, Real-time ASL recognition from video using Hidden Markov Models, in: ISCV, 1995.

[10] M. Brand, N. Oliver, A. Pentland, Coupled Hidded Markov models for complex action recognition, in: IEEE International Conference on Computer Vision and Pattern Recognition, 1996.

[11] C. Bregler, Learning and recognizing human dynamics in video sequences, in: IEEE International Conference on Computer Vision and Pattern Recognition, 1997.

[12] S. Gong, T. Xing, Recognition of group activities using dynamic probabilistic networks, in: IEEE International Conference on Computer Vision, 2003.

[13] C. Vogler, D. Metaxas, A framework for recognizing the simultaneous aspects of ASL, in: Computer Vision and Image Understanding, 2001.

[14] Z. Manor, M. Irani, Event-based analysis of video, in: IEEE International Conference on Computer Vision and Pattern Recognition, 2001.

[15] C. Stauffer, E. Grimson, Learning patterns of activity using real-time tracking, IEEE Transactions on Pattern Analysis and Machine Intelligence.

[16] A. Vasilescu, Human motion signatures: analysis, synthesis, recognition, 2002.

[17] A. Efros, A. Berg, G. Mori, J. Malik, Recognizing action at a distance, in: IEEE International Conference on Computer Vision, 2003.

[18] A. Bobick, A. Wilson, A state based technique for the summarization and recognition of gesture, in: IEEE International Conference on Computer Vision, 1995.

[19] A. Bobick, J. Davis, The recognition of human movement using temporal templates, in: IEEE Transactions on Pattern Analysis and Machine Intelligence, 2001.

[20] C. Pinhanez, A. Bobick, Human action detection using pnf propagation of temporal constraints, in: IEEE International Conference on Computer Vision and Pattern Recognition, 1998.

[21] S. Hongeng, R. Nevatia, F. Bremond, Video-based event recognition: activity representation and probabilistic recognition methods, Computer Vision and Image Understanding. 
[22] N. Olivier, E. Horovitz, A. Garg, Layered representations for human activity recognition, in: IEEE International Conference on Multimodal Interfaces, 2002.

[23] D. Ramanan, D. Forsyth, Automatic annotation of everyday movements, in: Advances in Neural Information Processing Systems, 2003.

[24] D. Zhang, D. Gatica-Perez, S. Bengio, I. McCowan, G. Lathoud, Modeling individual group actions in meetings: a two-layer HMM framewok, in: IEEE CVPR Workshop on Detection and Recognition of events in video, 2004.

[25] Z. Ghahramani, M. Jordan, Factorial Hidden Markov Models, Machine Learning.

[26] A. Blake, B. North, M. Isard, Learning Multi-Class Dynamics, Advances in Neural Information Processing Systems 11 (1999) 389-395.

[27] V. Pavlovic, J. Rehg, Imact of dynamic model learning on the classification of human motion, in: IEEE International Conference on Computer Vision and Pattern Recognition, 2000.

[28] M. Black, A. Jepson, A probabilistic framework for matching temporal trajectories: Condensation-based recognition of gestures and expressions, in: European Conference on Computer Vision, 1998.

[29] Y. Shi, Y. Huang, D. Minnen, A. Bobick, I. Essa, Propagation networks for recognition of partially ordered sequential action, in: IEEE International Conference on Computer Vision and Pattern Recognition, 2004.

[30] C. Fanti, L. Zelnik-Manor, P. Perona, Hybrid models for human motion recognition, in: IEEE International Conference on Computer Vision, 2005.

[31] H. J, J. Little, Representation and recognition of complex human motion, in: IEEE International Conference on Computer Vision and Pattern Recognition, 2000 .

[32] A. Yilmaz, M. Shah, Recognizing human actions in videos acquired by uncalibrated moving cameras, in: IEEE International Conference on Computer Vision, 2005.

[33] S. Kumar, M. Hebert, Discriminative random fields: A discriminative framework for contextual interaction and classification, in: IEEE International Conference on Computer Vision, 2003.

[34] X. He, R. Zemel, M. Carreira-Perpinan, Multiscale conditional random fields for image labeling, in: IEEE International Conference on Computer Vision and Pattern Recognition, 2004.

[35] A. Torralba, K. Murphy, W. Freeman, Contextual models for object detection using boosted random fields, in: Advances in Neural Information Processing Systems, 2004.

[36] A. Quattoni, M. Collins, T. Darrell, Conditional random fileds for object recognition, in: Advances in Neural Information Processing Systems, 2004. 
[37] N. Quian, T. Sejnowsky, Predicting the secondary structure of globular proteins using neural network models, J. Mol. Bio.

[38] M. Jordan, Learning in graphical models, MIT Press, 1998.

[39] A. McCallum, Efficiently inducing features of conditional random fields, in: Uncertainty in Artificial Intelligence, 2003.

[40] CMU Human Motion DataBase, Online at http://mocap.cs.cmu.edu/search.html (2003).

[41] C. Sminchisescu, A. Kanaujia, Z. Li, D. Metaxas, Discriminative Density Propagation for 3D Human Motion Estimation, in: IEEE International Conference on Computer Vision and Pattern Recognition, Vol. 1, 2005, pp. 390-397.

[42] C. Sminchisescu, A. Jepson, Variational Mixture Smoothing for Non-Linear Dynamical Systems, in: IEEE International Conference on Computer Vision and Pattern Recognition, Vol. 2, Washington D.C., 2004, pp. 608-615.

[43] C. Sminchisescu, A. Kanaujia, Z. Li, D. Metaxas, Conditional models for contextual human motion recognition, in: IEEE International Conference on Computer Vision, Vol. 2, 2005, pp. 1808-1815.

[44] C. Sminchisescu, A. Jepson, Generative Modeling for Continuous Non-Linearly Embedded Visual Inference, in: International Conference on Machine Learning, Banff, 2004, pp. 759-766.

[45] R. Urtasun, D. Fleet, A. Hertzmann, P. Fua, Priors for people tracking in small training sets, in: IEEE International Conference on Computer Vision, 2005. 